\title{
A juvenile cf. Edmontosaurus annectens (Ornithischia, Hadrosauridae) femur documents a previously unreported intermediate growth stage for this taxon
}

\author{
Andrew A. Farke $e^{1,2,3, *}$ and Eunice Yip ${ }^{2}$ \\ ${ }^{1}$ Raymond M. Alf Museum of Paleontology, 1175 West Baseline Road, Claremont, CA, 91711, USA \\ ${ }^{2}$ The Webb Schools, 1175 West Baseline Road, Claremont, CA, 91711, USA \\ ${ }^{3}$ Dinosaur Institute, Natural History Museum of Los Angeles County, 900 Exposition Blvd. Los Angeles, CA, \\ 90007, USA; afarke@webb.org
}

\begin{abstract}
A nearly complete, but isolated, femur of a small hadrosaurid from the Hell Creek Formation of Montana is tentatively referred to Edmontosaurus annectens. At $28 \mathrm{~cm}$ long, the element can be classified as likely that from an 'early juvenile' individual, approximately $24 \%$ of the maximum known femur length for this species. Specimens from this size range and age class have not been described previously for E. annectens. Notable trends with increasing body size include increasingly distinct separation of the femoral head and greater trochanter, relative increase in the size of the cranial trochanter, a slight reduction in the relative breadth of the fourth trochanter, and a relative increase in the prominence of the cranial intercondylar groove. The gross profile of the femoral shaft is fairly consistent between the smallest and largest individuals. Although an ontogenetic change from relatively symmetrical to an asymmetrical shape in the fourth trochanter has been suggested previously, the new juvenile specimen shows an asymmetric fourth trochanter. Thus, there may not be a consistent ontogenetic pattern in trochanteric morphology. An isometric relationship between femoral circumference and femoral length is confirmed for Edmontosaurus. Overall ontogenetic trends in the femur of Edmontosaurus are concordant with patterns seen in other Hadrosauridae, supporting a fairly conserved pattern of development for this element within the clade.
\end{abstract}

\section{INTRODUCTION}

Ontogenetic changes in hadrosaurid dinosaurs are known in substantial detail for several species, supported by the study of isolated elements, articulated skeletons, and multi-individual bonebed assemblages. Documentation of gross morphology, osteohistology, and allometric trends permit inference of heterochrony, life history traits, functional changes, and other attributes both within species and across species. Within the lambeosaurine (hollow-crested) hadrosaurids, ontogenetic studies have focused primarily on development of the cranial ornamentation (e.g., Dodson 1975; Evans 2010), and to a lesser degree on postcranial ontogeny (e.g., Guenther 2009, 2014; Fondevilla et al. 2018) or both (e.g., Horner and Currie 1994; Farke et al. 2013). Among hadrosaurine hadrosaurids, postcranial

Published April 30, 2019

*corresponding author. $\odot 2019$ by the authors submitted Dec. 18, 2019; revisions received April 22, 2019; accepted April 25, 2019. Handling editor: Robert Holmes. DOI 10.18435/vamp29347 growth series are particularly broad (i.e., preserving individuals spanning a spectrum from very small and early in life to very large and presumably adult) for Maiasaura peeblesorum, Saurolophus angustirostrus, and Edmontosaurus annectens (Bell 2011; Guenther 2014; Prieto-Márquez 2014; Dewaele et al. 2015; Woodward et al. 2015; Wosik et al. 2017; Prieto-Marquez and Guenther 2018).

Edmontosaurus annectens is a common component of the faunal assemblage for the Hell Creek, Lance, Frenchman, and Scollard formations (latest Maastrichtian, -68-66.0 $\mathrm{Ma}$; for one recent summary of ages and correlations, see Fowler 2017) of western North America (Campione and Evans 2011). Cranial changes during ontogeny have received particular attention, due to their relevance to taxonomic problems. Postcranial changes are more sparsely documented. Although bonebed assemblages have the potential to document a range of body sizes for $E$. annectens, none of these have been formally described with respect to gross skeletal morphology (Colson et al. 2004; Stanton Thomas and Carlson 2004; Wosik 2018). Instead, most information on postcranial ontogenetic changes in $E$. annectens is based upon exceptionally preserved articu- 
lated individuals, particularly for the first half of ontogeny. Most notably, Prieto-Marquez (2014) described a nearly complete skeleton from a late juvenile-sized individual ( $-47 \%$ of maximum known femur length for the taxon). This was followed by Wosik et al.'s description (2017) of the partial articulated postcranium from a nestling-sized individual $(-12 \%$ of maximum known femur length for E. annectens). Despite the reputation of E. annectens as a common fossil, the postcrania are still rather sparsely described for most size classes. Similarly, described postcranial elements from the closely related Edmontosaurus regalis and Shantungosaurus giganteus are for individuals at 50\% or more of maximum known element length (Baert et al. 2014; Hone et al. 2014; Vanderven et al. 2014). Postcrania for Ugrunaaluk kuukpikensis are around 36 percent of maximum size for Edmontosaurus annectens (Mori et al. 2015), but have not been described in detail; note that this taxon is currently of uncertain status (Xing et al. 2014, 2017). The femur has figured prominently in studies of dinosaurian ontogeny (and hadrosaurid ontogeny) for multiple reasons. First, it is a robust and frequently well preserved element with morphology that permits confident assignment to the family (and often subfamily) level when found in isolation. Additionally, the femur is an attachment site for major muscles that function in locomotion, so it is useful for understanding functional changes during ontogeny (e.g., Dilkes 2001). Finally, as a major load bearing bone, it can be used (along with the humerus) to estimate the biologically informative parameter of body mass (e.g., Campione and Evans 2012; Campione 2014; Wosik et al. 2017), which also is functionally linked with inferred locomotor changes during ontogeny (e.g., Dilkes 2001; Wosik et al. 2017). Thus, detailed study of this element is particularly worthwhile.

Here, we describe an isolated femur (RAM 9396) identified as an early juvenile Edmontosaurus annectens from the Hell Creek Formation of Montana. At approximately 28 $\mathrm{cm}$ long, it falls within a size gap between the previously described smallest (UCMP 128181, $15 \mathrm{~cm}$ long, at the "late nestling" ontogenetic stage, sensu Wosik et al. 2018) and next smallest (LACM 23504, $56 \mathrm{~cm}$ long, at the "late juvenile" ontogenetic stage, sensu Wosik et al. 2018) femora. Accordingly, RAM 9396 provides new information on ontogenetic changes in E. annectens, as well as data relevant to ontogenetic comparisons across hadrosaurids.

\section{Institutional abbreviations}

AMNH, American Museum of Natural History, New York, New York, USA; CCM, Carter County Museum, Ekalaka, Montana, USA; CMN, Canadian Museum of Nature, Ottawa, Ontario, Canada; CMNH, Cleveland Museum of Natural History, Cleveland, Ohio, USA;
DMNH, Denver Museum of Nature and Science, Denver, Colorado, USA; FPDM, Fukui Prefectural Dinosaur Museum, Katsuyama, Japan; LACM, Natural History Museum of Los Angeles County, Los Angeles, California, USA; MOR, Museum of the Rockies, Bozeman, Montana, USA; RAM, Raymond M. Alf Museum of Paleontology, Claremont, California, USA; ROM, Royal Ontario Museum, Toronto, Ontario, Canada; SDSM, Museum of Geology, South Dakota School of Mines and Technology, Rapid City, South Dakota, USA; UALVP, University of Alberta Laboratory of Vertebrate Paleontology, Calgary, Alberta, Canada; UCMP, University of California Museum of Paleontology, Berkeley, California, USA; USNM, National Museum of Natural History, Washington, D.C., USA.

\section{Ontogenetic terminology}

Here, we use terminology for ontogenetic stages as defined by Wosik et al. (2017), which was modified in turn from that of Horner et al. (2000). Because RAM 9396 was not sectioned for histological study, stage referral primarily is based upon element length. Presumed incomplete ossification of bone ends and porous surface texture on the femoral shaft are also consistent with non-adult status (e.g., Horner et al. 2000; Tumarkin-Deratzian et al. 2006; Wosik 2018), but cannot be used to refine the age class more precisely.

\section{Geological background}

RAM 9396 was collected in 2000 by a team from the Raymond M. Alf Museum of Paleontology and The Webb Schools, at RAM locality V200029. This site is within the basal lag of a channel sandstone situated within the upper part of the Hell Creek Formation of McCone County, Montana, USA. Although RAM 9396 was collected in situ, no other bones referable to this individual were recovered at the site. Detailed locality data are on file at RAM, available upon request.

\section{Taxonomic referral}

Because the Hell Creek Formation yields numerous tetrapod taxa that have femora within the size range of RAM 9396, we briefly justify our tentative assignment of this specimen to Edmontosaurus annectens. Overall shape of the specimen (e.g., comparatively straight diaphysis and prominent fourth trochanter) excludes identification as a turtle, choristodere, or crocodyliform, and the solid cross-section observed during conservation of the fossil further excludes assignment to a pterosaur or theropod. The non-pendant fourth trochanter differs from that seen in "basal" ornithopods with femora of smaller or roughly similar size such as Thescelosaurus (Brown et al. 2011), pachycephalosaurids (Gilmore 1924), and neoceratopsians such as Leptoceratops 
(Brown 1914). Furthermore, the fourth trochanter is more pronounced than seen in ceratopsids (e.g., Triceratops) or ankylosaurians. Although small femora have not been described for Triceratops, those from juveniles, subadults, and adults of other ceratopsids are characterized by a fourth trochanter that is more proximally placed on the femur relative to the condition in RAM 9396 and Hadrosauridae (e.g., Lehman 1989). The shafts of ankylosaur femora for individuals of various sizes tend to be much more cranio-caudally compressed than seen in hadrosaurids or RAM 9396, and ankylosaur femora are overall more "robust" (relatively broader shaft relative to length) than hadrosaurid femora even at small size (Carpenter 2004; Currie et al. 2011; Zheng et al. 2018). Thus, in overall form, major aspects of the femoral anatomy in RAM 9396 match those seen in much larger Edmontosaurus annectens (as outlined below). Although the femur itself does not hold autapomorphies that permit assignment to the species or even genus level, E. annectens is currently the only valid hadrosaurid known from the Hell Creek Formation (Campione and Evans 2011). Thus, we tentatively refer RAM 9396 to this taxon.

\section{DESCRIPTION}

RAM 9396 is a nearly complete left femur, missing only the posterior parts of the distal condyles (Figs. 1, 2). It has a maximum length of $277 \mathrm{~mm}$, as measured along the lateral surface; comprehensive measurements are illustrated in Figure 3 and provided in Table 1. Here, we describe the element beginning with the proximal end and compare RAM 9396 with other specimens of Edmontosaurus spp.
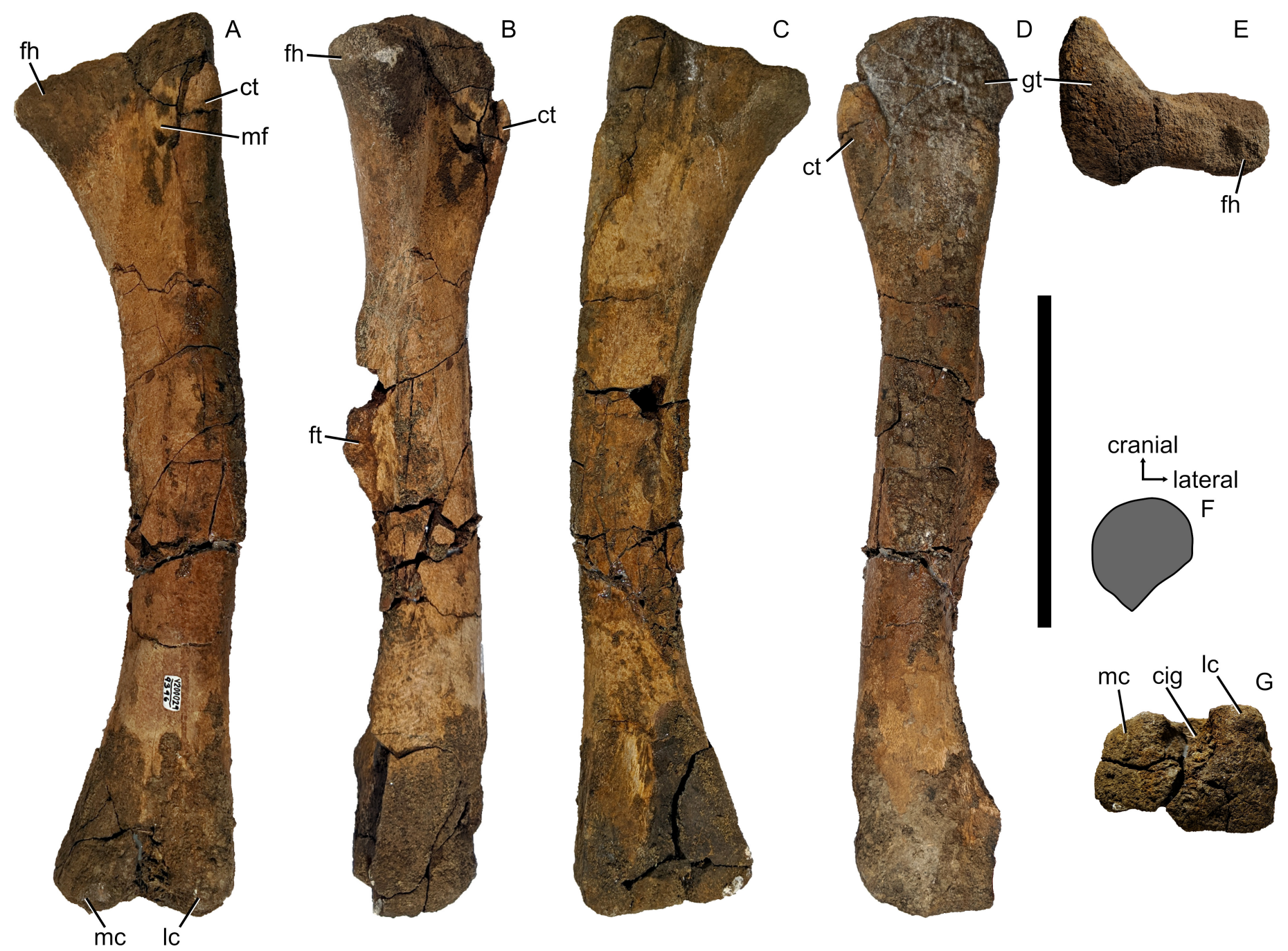

Figure 1. RAM 9396, left femur of cf. Edmontosaurus annectens, photographed in A) anterior; B) medial; C) posterior; D) lateral; E) proximal; F) diaphyseal cross-section with directional arrows, just distal to the fourth trochanter and approximately at the point of measurement 4 in Figure 3B; and G) distal views. Scale bar equals $10 \mathrm{~cm}$. Abbreviations: cig, cranial intertrochanteric groove; ct, cranial trochanter; fh, femoral head; ft, fourth trochanter; gt, greater trochanter; Ic, lateral condyle; $\mathrm{mc}$, medial condyle; mf, muscular fossa. 

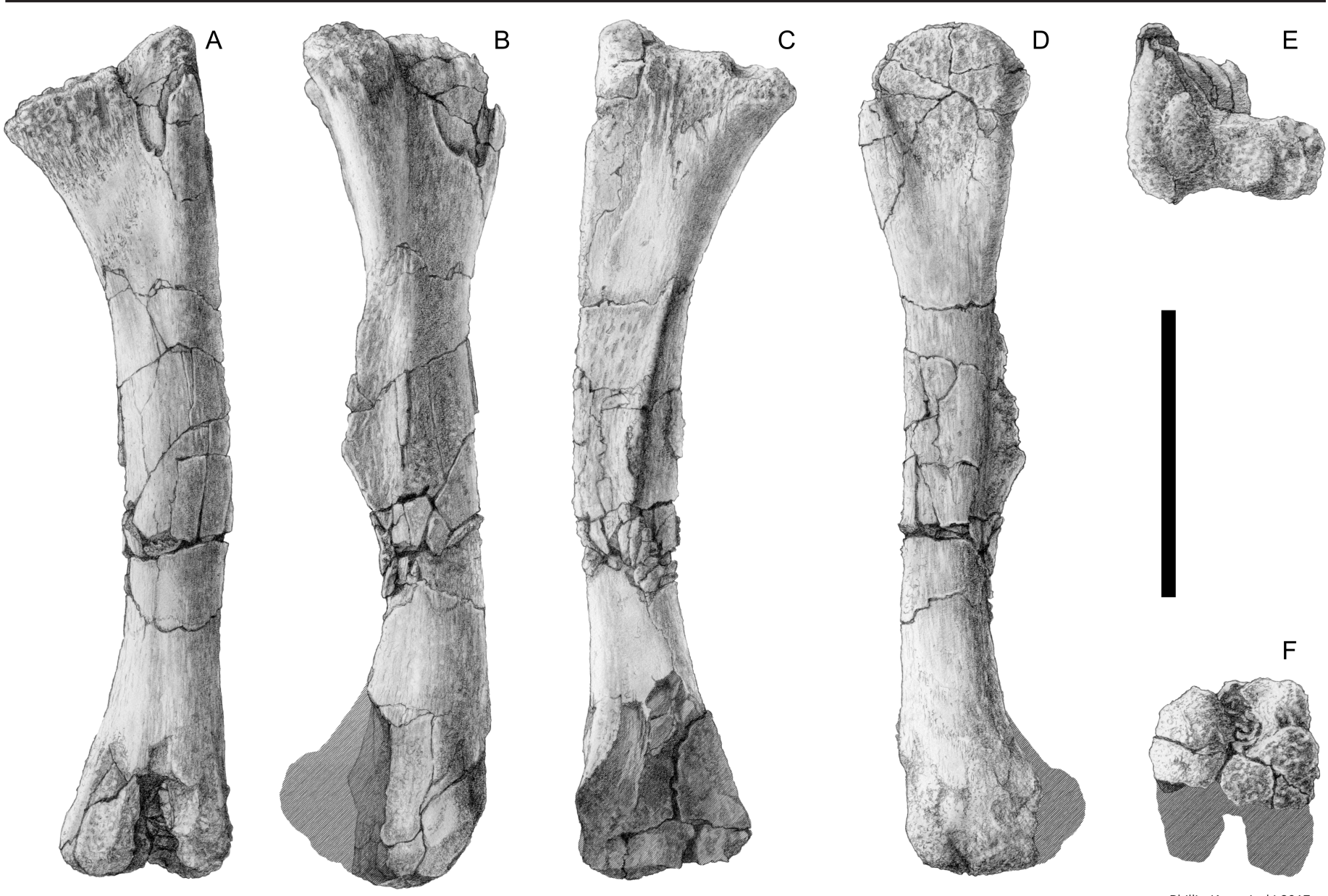

Figure 2. RAM 9396, left femur of cf. Edmontosaurus annectens, drawn in A) anterior; B) medial; C) posterior; D) lateral; E) proximal; and F) distal views. Missing portions of the condyles are indicated with gray shading. Scale bar equals $10 \mathrm{~cm}$. Illustrated by Phillip Krzeminski, used with permission.

In dorsal view the femoral head of RAM 9396 is roughly rectangular and set at a $90^{\circ}$ angle relative to the greater trochanter, which is roughly triangular (Figs. 1E, 2E). In larger individuals of Edmontosaurus spp., the two structures are separated in dorsal view by a distinct constriction of the bone on both its cranial and caudal surfaces (e.g., LACM 23504; also see fig. 13.20F, L in Campione 2014). In RAM 9396, the constriction is much less pronounced, and the head and greater trochanter are less distinctly separated (Figs. 1E, 2E). The bone surface in this region is gently abraded, so it is not possible to gauge the extent of cartilaginous surfaces; we note that this may also affect the preserved shape of the proximal femur. This abrasion may be due in part to incomplete ossification, related to the young age of the individual. When the main body of the femoral shaft is oriented vertically and viewed cranially (Figs. 1A, 2A), the femoral head is set slightly ventrally (distally) relative to the greater trochanter, and the two are separated by a slight depression. The condition in larger individuals is hard to assess due to crushing in many specimens. For instance, the head is ventral relative to the greater trochanter in the subadult $E$. annectens LACM 23504 and in the left femur of the large E. regalis individual CMN 2289, but at nearly the same level in the right femur of CMN 2289. In lateral view (Figs. 1D, 2D), the dorsal (proximal) end of the greater trochanter on RAM 9396 has a semicircular profile, comparable in shape and proportions to the greater trochanter in larger juvenile (LACM 23504) and adult-sized (AMNH 5886, USNM 2414 ) individuals of $E$. annectens. The tab-like cranial trochanter is well defined and placed ventral to the greater trochanter, at approximately the same level as the femoral head (Figs. 1A, 2A). This cranial trochanter of RAM 9396 is proportionately smaller than the condition in larger individuals; in large Edmontosaurus, the cranial trochanter sometimes approaches the same width as the greater trochanter (e.g., CMN 2289; AMNH 5886), although it may also be smaller (USNM 2414). A distinctly rimmed fossa immediately medial to the cranial trochanter occurs on the cranial surface of the femur (Fig. 1A), which may represent the insertion for $\mathrm{m}$. iliofemoralis (following muscle maps from Maidment et al. 2014). 

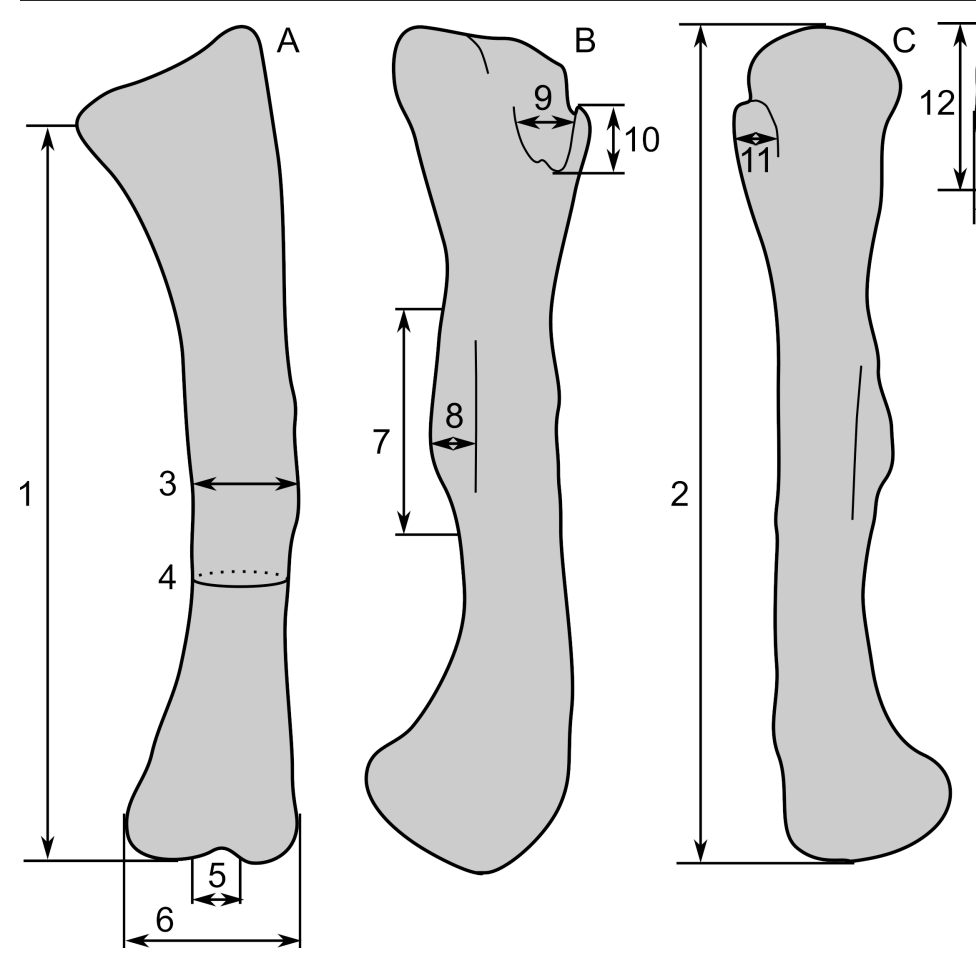

Figure 3. Measurement parameters for RAM 9396, left femur of cf. Edmontosaurus annectens, drawn in A) anterior; B) medial; C) lateral; and D) proximal views. Numbers correspond with measurements in Table 1.
The femoral shaft of RAM 9396 is fairly straight along its longitudinal axis in lateral view (Figs. 1D, 2D). In cranial view (Figs. 1A, 2A), the lateral surface is slightly bowed convexly, and the medial surface has an even more strongly concave profile relative to the long axis. Thus, the overall conformation of the shaft matches that seen in smaller and larger Edmontosaurus (e.g., UCMP 128181; LACM 23504). The fourth trochanter is centered at approximately midshaft (comparable with individuals across all sizes in Edmontosaurus annectens) and occupies just over a quarter of the length of the femur along its caudomedial surface
(Figs. 1B, 2B). The trochanter occupies a proportionately smaller percentage of the femoral shaft length in larger individuals, around $20 \%$ of the shaft length in the juvenile LACM 23504 and adult AMNH 5886. The trochanter has a broad-based, asymmetrically triangular profile, with the tip of the triangle placed distally relative to the center of the triangle (Fig. 4A). The base of the trochanter is relatively narrower in some larger individuals (Fig. 4B, C), which creates a more prominent appearance for the structure. The outline is also asymmetrical in some large individuals (Prieto-Márquez 2014; Wosik et al. 2017), but this varies

Table 1. Measurements of RAM 9396, left femur of cf. Edmontosaurus annectens. Measurement standards are illustrated in Figure 3

\section{Description}

Maximum length, medial surface

2 Maximum length, lateral surface

3 Medio-lateral width at midshaft

4 Circumference of shaft, just below fourth trochanter

5 Distance between cranial edges of medial and lateral distal condyles

6 Maximum width of distal end

7 Maximum length of fourth trochanter

8 Maximum height of fourth trochanter

9 Maximum width of ?iliofemoralis insertion fossa

10 Maximum proximo-distal length of cranial trochanter

11 Maximum cranio-caudal length of cranial trochanter

12 Maximum cranio-caudal length of proximal end

13 Maximum medio-lateral width of proximal end

14 Maximum cranio-caudal width of femoral head
Measurement (mm)

252.0

277.1

35.9

105.0

11.7

55.5

76.2

11.4

20.5

24.4

16.8

48.1

64.1

24.8 


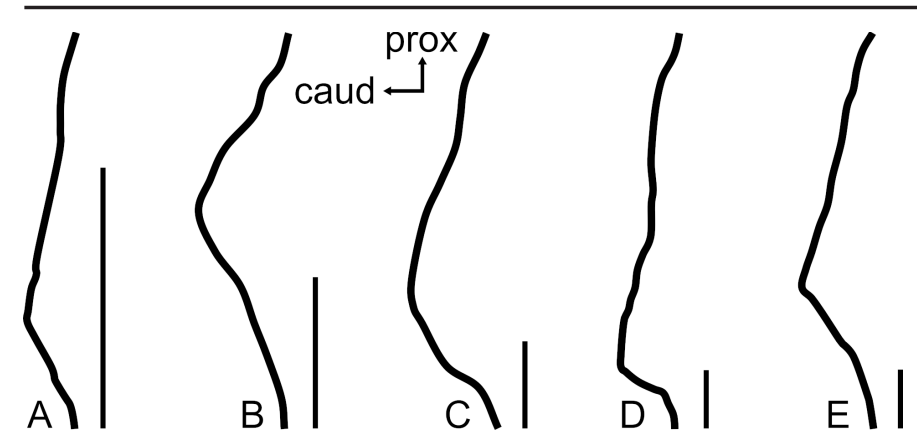

Figure 4. Comparative line drawings of the fourth trochanter from Edmontosaurus annectens, showing variation in the proximo-distal position of the trochanter's apex. The arrow diagram indicates proximal (prox) and caudal (caud) directions. Specimens are arranged from smallest (left) to largest trochanter (right), for A) RAM 7967; B) LACM 23504; C) USNM 2414; D) RAM 318; E) RAM 7150. Scale bar equals $5 \mathrm{~cm}$.

between specimens, possibly due to taphonomic effects. For instance, the greater trochanters of large $E$. annectens (AMNH 5886, RAM 318, RAM 7150, and USNM 2414; Fig. 4C, D, E) are asymmetric with the apex positioned distally, whereas the same structure is fairly symmetrical in the large E. regalis CMN 2289 and the subadult $E$. annectens LACM 23504 (Fig. 4B). A slight concavity occupies most of the medial surface of the fourth trochanter in RAM 9396, which presumably represents the insertion for the m. caudofemoralis group (Dilkes 1999; Maidment et al. 2014). The femoral shaft of RAM 9396 is uncrushed and has a teardrop-shaped cross-section at mid-shaft (when the trochanter is ignored), with the caudo-lateral surface broadest and slightly flattened (Fig. 1F). Because this region was crushed in the other specimens we examined, we cannot compare the cross-sectional shape of RAM 9396 with that in larger individuals.

In distal view, the medial condyle in RAM 9396 is slightly more prominent than the lateral condyle, and they are separated by a prominent cranial intercondylar groove (Figs. 1G, 2F); both conditions match that seen in larger Edmontosaurus annectens (e.g., RAM 318, 7150). One distinct difference between RAM 9396 and larger Edmontosaurus (LACM 23504, AMNH 5886, USNM 2414) is that larger individuals sometimes have a slight cranial protrusion of the condyles. With the lateral surface of the shaft oriented vertically, the lateral condyle is in approximately the same vertical plane as the greater trochanter, and the medial condyle is very slightly lateral to the vertical plane that contains the femoral head (Figs. $1 \mathrm{~A}, 2 \mathrm{~A})$, matching the condition in larger individuals. A slightly depressed area, perhaps representing the attachment site of gastrocnemius and associated muscles (Dilkes 1999; Maidment et al. 2014), occurs along the surface of the lateral condyle and onto the cranio-proximal surface of the diaphysis. The proximal surfaces of the condyles are slightly abraded, which may reflect reduced ossification relative to other parts of the femur. The caudal surfaces of the distal condyles and the associated caudal intercondylar groove are not preserved.

\section{STATISTICAL ANALYSIS}

Due to limitations in the available sample, a previous analysis of femoral allometry in Edmontosaurus was heavily skewed towards large individuals (Wosik et al. 2017), which can hamper interpretations of allometry. Thus, RAM 9396 was incorporated into the dataset of Wosik et al. (2017), regressing femoral circumference onto femoral length. Two additional femora (UALVP 50988 and 52758) from a disarticulated, multi-taxon (but monodominant) bonebed assemblage referable to $E$. regalis were added to the sample, using published measurements (Baert et al. 2014; Vanderven et al. 2014). Measurements were log-transformed with a natural log function, and then analyzed with RMA (reduced major axis) regression. Regressions were calculated within PAST 3.22 (Hammer et al. 2001), using the full sample with both $E$. annectens and $E$. regalis as well as a subsample with just $E$. annectens.

The new regression (Fig. 5) had a slope indistinguishable from isometry when considering all Edmontosaurus (1.041; $95 \%$ confidence interval of $0.7802-1.079 ; \mathrm{r} 2=0.96 ; \mathrm{n}=21)$ and when including just $E$. annectens $(1.0183 ; 95 \%$ confidence interval of $0.407-1.087 ; \mathrm{r} 2=0.98 ; \mathrm{n}=15)$. These results do not differ significantly from the finding of isometry by Wosik et al. (2017).

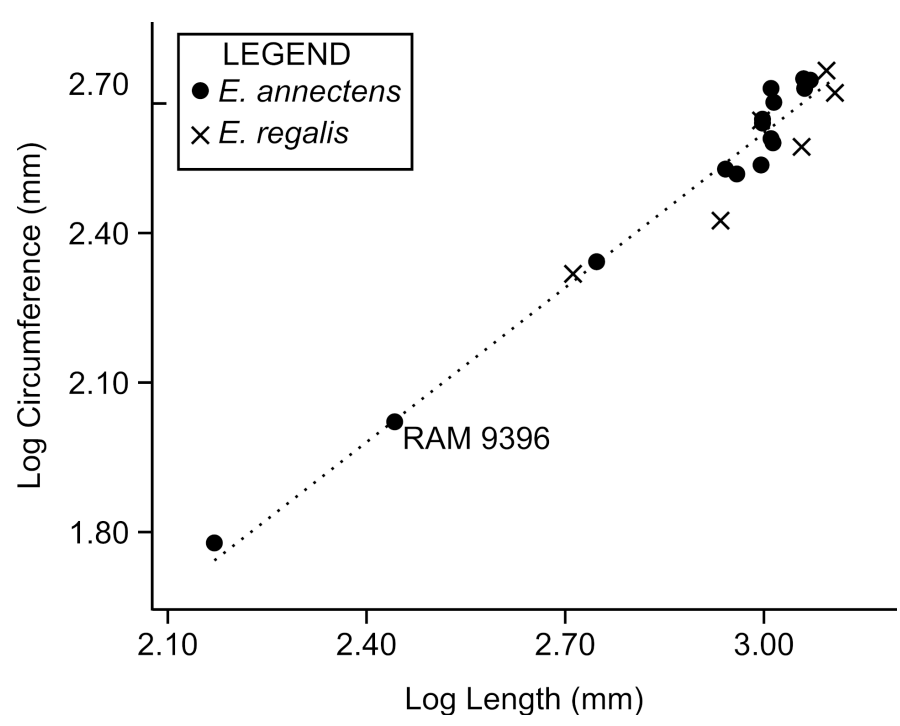

Figure 5. Linear regression (RMA) showing femur length versus femur circumference for Edmontosaurus spp. Data presented in Table 2. 


\section{BODY MASS ESTIMATE}

We used Developmental Mass Extrapolation (Erickson and Tumanova 2000) to estimate the body mass of RAM 9396. Following the assumptions of Wosik et al. (2017), we used CCM V 1938.8 specimen for comparison. Depending on the metric used for scaling, body mass for RAM 9396 is estimated as $68 \mathrm{~kg}$ (when scaled by femoral circumference) or $96 \mathrm{~kg}$ (when scaled by femoral length).

\section{DISCUSSION}

The description and comparisons of a femur referred to a small juvenile Edmontosaurus annectens help to clarify both previously documented and previously unrecognized patterns in the ontogeny of the femur in this taxon. Major changes with increasing body size include a more distinct separation of the femoral head and greater trochanter (at subadult sizes; e.g., LACM 23504), relative increase in the size of the cranial trochanter (variable, but seen most commonly in adult sizes; e.g., AMNH 5886), a slight reduction in the relative size of the fourth trochanter (occurring at subadult sizes; e.g., LACM 23504), and a relative increase in the prominence of the cranial intercondylar groove (occurring at full adult size). The gross profile of the femoral shaft is fairly consistent between the smallest and largest individuals. Although a major shift from relatively symmetrical to asymmetrical shape in the fourth trochanter has been described in the literature (also noted by PrietoMárquez 2014; Wosik et al. 2017), the asymmetric shape in the relatively small RAM 9396 indicates that this is probably not a clear trend, but instead may just reflect individual variation in the shape of the trochanter independent of ontogeny (Fig. 4).

Inclusion of RAM 9396 in an allometric analysis of femoral proportions in Edmontosaurus does not change previous interpretations of isometry in femoral circumference versus length in this animal (Wosik et al. 2017). Nonetheless, the fossil is important for filling a size gap in the dataset (Table 2 and Fig. 5); the collection and measurement of other specimens will undoubtedly help to firm up interpretations of femoral allometry for Edmontosaurus. Based on the overall sample in the literature, the general ontogenetic trajectory for femora in Edmontosaurus is consistent with that described for other hadrosaurines. Femoral ontogeny was quite similar in Maiasaura and Edmontosaurus (Dilkes 2001; Guenther 2014; PrietoMarquez and Guenther 2018), and indeed even similar to that in the lambeosaurine Hypacrosaurus (Guenther 2014). Thus, overall ontogenetic trends for the femur were probably conserved across Hadrosauridae, as noted previously (Brett-Surman and Wagner 2006; Guenther 2009, 2014).

\section{ACKNOWLEDGEMENTS}

We thank Gabe Santos and Lucy Herrero for collections assistance and Jared Heuck for preparation assistance. Joe Sertich (DMNH) and Maureen Walsh (LACM) facilitated access to specimens under their care. Discussions with Mateusz Wosik and Don Lofgren were helpful in understanding this fossil and its broader context. Phillip Krzeminski skillfully prepared the artistic renderings of RAM 9396 in Figure 2. E. Yip's participation in this work was supported by the David B. Jones Foundation and the Mary Stuart Rogers Foundation, with additional support for the project from the Augustyn Family Endowment and Research Fund. RAM 9396 was collected under Paleontology Special Use Permit 00-10 from Charles M. Russell National Wildlife Refuge, Fish and Wildlife Service, United States Department of the Interior. Comments from Mateusz Wosik and an anonymous reviewer were helpful in improving the manuscript during review, and we also acknowledge editor Rob Holmes for his able assistance.

\section{LITERATURE CITED}

Baert, M., M.E. Burns, and P.J. Currie. 2014. Quantitative diagenetic analyses of Edmontosaurus regalis (Dinosauria: Hadrosauridae) postcranial elements from the Danek Bonebed, Upper Cretaceous Horseshoe Canyon Formation, Edmonton, Alberta, Canada: implications for allometric studies of fossil organisms. Canadian Journal of Earth Sciences 51:1007-1016.

Bell, P.R. 2011. Cranial osteology and ontogeny of Saurolophus angustirostris from the Late Cretaceous of Mongolia with comments on Saurolophus osborni from Canada. Acta Palaeontologica Polonica 56:703-722.

Brett-Surman, M.K., and J.R. Wagner. 2006. Discussion of character analysis of the appendicular anatomy in Campanian and Maastrichtian North American hadrosaurids - variation and ontogeny. Pp. 135-169 in K. Carpenter (ed.). Horns and Beaks: Ceratopsian and Ornithopod Dinosaurs. Indiana University Press, Bloomington.

Brown, B. 1914. Leptoceratops, a new genus of Ceratopsia from the Edmonton Cretaceous of Alberta. Bulletin of the American Museum of Natural History 33:567-580.

Brown, C.M., C.A. Boyd, and A.P. Russell. 2011. A new basal ornithopod dinosaur (Frenchman Formation, Saskatchewan, Canada), and implications for late Maastrichtian ornithischian diversity in North America. Zoological Journal of the Linnean Society 163:1157-1198.

Campione, N.E. 2014. Postcranial anatomy of Edmontosaurus regalis (Hadrosauridae) from the Horseshoe Canyon Formation, Alberta, Canada. Pp. 208-244 in D.A Eberth and D.C. Evans (eds.). Hadrosaurs. Proceedings of the International Hadrosaur Symposium. Indiana University Press, Bloomington.

Campione, N.E., and D.C. Evans. 2011. Cranial growth and variation in edmontosaurs (Dinosauria: Hadrosauridae): impli- 
cations for latest Cretaceous megaherbivore diversity in North America. PLOS ONE 6:e25186.

Campione, N.E., and D.C. Evans. 2012. A universal scaling relationship between body mass and proximal limb bone dimensions in quadrupedal terrestrial tetrapods. BMC Biology 10:60.

Carpenter, K. 2004. Redescription of Ankylosaurus magniventris Brown 1908 (Ankylosauridae) from the Upper Cretaceous of the Western Interior of North America. Canadian Journal of Earth Sciences 41:961-986.

Colson, M.C., R.O. Colson, and R. Nellermoe. 2004. Stratigraphy and depositional environments of the upper Fox Hills and lower Hell Creek Formations at the Concordia Hadrosaur Site in northwestern South Dakota. Rocky Mountain Geology 39:93-111.

Currie, P.J., D. Badamgarav, E.B. Koppelhus, R.Sissons, and M.K. Vickaryous. 2011. Hands, feet, and behaviour in Pinacosaurus (Dinosauria: Ankylosauridae). Acta Palaeontologica Polonica 56:489-504.

Dewaele, L., K. Tsogtbaatar, R. Barsbold, G. Garcia, K. Stein, F. Escuillié, and P. Godefroit. 2015. Perinatal specimens of Saurolophus angustirostris (Dinosauria: Hadrosauridae), from the Upper Cretaceous of Mongolia. PLOS ONE 10:e0138806.

Dilkes, D.W. 1999. Appendicular myology of the hadrosaurian dinosaur Maiasaura peeblesorum from the Late Cretaceous (Campanian) of Montana. Earth and Environmental Science Transactions of the Royal Society of Edinburgh 90:87-125.

Dilkes, D.W. 2001. An ontogenetic perspective on locomotion in the Late Cretaceous dinosaur Maiasaura peeblesorum (Ornithischia: Hadrosauridae). Canadian Journal of Earth Sciences 38:1205-1227.

Dodson, P. 1975. Taxonomic implications of relative growth in lambeosaurine hadrosaurs. Systematic Zoology 24:37-54.

Erickson, G.M., and T.A. Tumanova. 2000. Growth curve of Psittacosaurus mongoliensis Osborn (Ceratopsia: Psittacosauridae) inferred from long bone histology. Zoological Journal of the Linnean Society 130:551-566.

Evans, D.C. 2010. Cranial anatomy and systematics of Hypacrosaurus altispinus, and a comparative analysis of skull growth in lambeosaurine hadrosaurids (Dinosauria: Ornithischia). Zoological Journal of the Linnean Society 159:398-434.

Farke, A.A., D.J. Chok, A. Herrero, B. Scolieri, and S. Werning. 2013. Ontogeny in the tube-crested dinosaur Parasaurolophus (Hadrosauridae) and heterochrony in hadrosaurids. PeerJ $1: e 182$.

Fondevilla, V., F.M.D. Vecchia, R. Gaete, À. Galobart, B. Moncunill-Solé, and M. Köhler. 2018. Ontogeny and taxonomy of the hadrosaur (Dinosauria, Ornithopoda) remains from Basturs Poble bonebed (late early Maastrichtian, Tremp Syncline, Spain). PLOS ONE 13:e0206287.

Fowler, D.W. 2017. Revised geochronology, correlation, and dinosaur stratigraphic ranges of the Santonian-Maastrichtian
(Late Cretaceous) formations of the Western Interior of North America. PLOS ONE 12:e0188426.

Gilmore, C.W. 1924. On Troodon validus, an orthopodous dinosaur from the Belly River Cretaceous of Alberta, Canada. University of Alberta, Department of Geology, Bulletin 1:1-43.

Guenther, M.F. 2009. Influence of sequence heterochrony on hadrosaurid dinosaur postcranial development. The Anatomical Record 292:1427-1441.

Guenther, M.F. 2014. Comparative ontogenies (appendicular skeleton) for three hadrosaurids and a basal iguanodontian: divergent developmental pathways in Hadrosaurinae and Lambeosaurinae. Pp. 398-415 in D.A. Eberth and D.C. Evans (eds.). Hadrosaurs. Indiana University Press, Bloomington.

Hammer, Ø., D.A.T. Harper, and P.D. Ryan. 2001. Past: Paleontological Statistics Software Package for education and data analysis. Palaeontologia Electronica 4:1-9.

Hone, D.W.E., C. Sullivan, Q. Zhao, K. Wang, and X. Xu. 2014. Body size distribution in a colossal hadrosaurid death assemblage from the Upper Cretaceous of Zhucheng, Shandong Province, China. Pp. 524-531 in D.A. Eberth and D.C. Evans (eds.). Hadrosaurs. Indiana University Press, Bloomington.

Horner, J.R., and P.J. Currie. 1994. Embryonic and neonatal morphology and ontogeny of a new species of Hypacrosaurus (Ornithischia, Lambeosauridae) from Montana and Alberta. Pp. 312-336 in K. Carpenter (ed.). Dinosaur Eggs and Babies. Cambridge University Press, Cambridge.

Horner, J.R., A. de Ricqlès, and K. Padian. 2000. Long bone histology of the hadrosaurid dinosaur Maiasaura peeblesorum: growth dynamics and physiology based on an ontogenetic series of skeletal elements. Journal of Vertebrate Paleontology 20:115-129.

Lehman, T. M. 1989. Chasmosaurus mariscalensis, sp. nov., a new ceratopsian dinosaur from Texas. Journal of Vertebrate Paleontology 9:137-162.

Maidment, S.C.R., K.T. Bates, and P.M. Barrett. 2014. Three-dimensional computational modeling of pelvic locomotor muscle moment arms in Edmontosaurus (Dinosauria, Hadrosauridae) and comparisons with other archosaurs. Pp. 433-448 in D.A. Eberth and D.C. Evans (eds.). Hadrosaurs: Proceedings of the International Hadrosaur Symposium. Indiana University Press, Bloomington.

Mori, H., P.S. Druckenmiller, and G.M. Erickson. 2015. A new Arctic hadrosaurid from the Prince Creek Formation (Lower Maastrichtian) of northern Alaska. Acta Palaeontologica Polonica 61:15-32.

Prieto-Márquez, A. 2014. A juvenile Edmontosaurus from the late Maastrichtian (Cretaceous) of North America: Implications for ontogeny and phylogenetic inference in saurolophine dinosaurs. Cretaceous Research 50:282-303.

Prieto-Marquez, A., and M.F. Guenther. 2018. Perinatal specimens of Maiasaura from the Upper Cretaceous of Montana (USA): insights into the early ontogeny of saurolophine hadrosaurid dinosaurs. PeerJ 6:e4734. 
Stanton Thomas, K.J., and S.J. Carlson. 2004. Microscale $\delta^{18} \mathrm{O}$ and $\delta^{13} \mathrm{C}$ isotopic analysis of an ontogenetic series of the hadrosaurid dinosaur Edmontosaurus: implications for physiology and ecology. Palaeogeography, Palaeoclimatology, Palaeoecology 206:257-287.

Tumarkin-Deratzian, A.R., D.R. Vann, and P. Dodson. 2006. Bone surface texture as an ontogenetic indicator in long bones of the Canada goose Branta canadensis (Anseriformes: Anatidae). Zoological Journal of the Linnean Society 148:133168.

Vanderven, E., M.E. Burns, and P.J. Currie. 2014. Histologic growth dynamic study of Edmontosaurus regalis (Dinosauria: Hadrosauridae) from a bonebed assemblage of the Upper Cretaceous Horseshoe Canyon Formation, Edmonton, Alberta, Canada. Canadian Journal of Earth Sciences 51:1023-1033.

Woodward, H.N., E.A. Freedman Fowler, J.O. Farlow, and J.R. Horner. 2015. Maiasaura, a model organism for extinct vertebrate population biology: a large sample statistical assessment of growth dynamics and survivorship. Paleobiology 41:503-527.

Wosik, M. 2018. Osteohistology and growth of Edmontosaurus and assessment of life history changes in hadrosaurid dinosaurs.
Ph.D. Dissertation, University of Toronto, Toronto, Ontario, Canada, 285 pp.

Wosik, M., M.B. Goodwin, and D.C. Evans. 2017. A nestling-sized skeleton of Edmontosaurus (Ornithischia, Hadrosauridae) from the Hell Creek Formation of northeastern Montana, U.S.A., with an analysis of ontogenetic limb allometry. Journal of Vertebrate Paleontology 37:e1398168.

Xing, H., J.C. Mallon, and M.L. Currie. 2017. Supplementary cranial description of the types of Edmontosaurus regalis (Ornithischia: Hadrosauridae), with comments on the phylogenetics and biogeography of Hadrosaurinae. PLOS ONE 12:e0175253.

Xing, H., X. Zhao, K. Wang, D. Li, S. Chen, J.C. Mallon, Y. Zhang, and X. Xu. 2014. Comparative osteology and phylogenetic relationship of Edmontosaurus and Shantungosaurus (Dinosauria: Hadrosauridae) from the Upper Cretaceous of North America and East Asia. Acta Geologica Sinica - English Edition 88:1623-1652.

Zheng, W., X. Jin, Y. Azuma, Q. Wang, K. Miyata, and X. Xu. 2018. The most basal ankylosaurine dinosaur from the AlbianCenomanian of China, with implications for the evolution of the tail club. Scientific Reports 8:3711. 